\title{
THE PRESENT STATUS OF THE JAERI/KEK JOINT PROJECT FOR HIGH-INTENSITY PROTON ACCELERATORS
}

\author{
Y. Yamazaki, KEK, Tsukuba-shi, Ibaraki-ken 305-0801, Japan \\ and JAERI, Tokai-mura, Naka-gun, Ibaraki-ken, 319-1195, Japan
}

\begin{abstract}
The high-intensity proton accelerator facility in Japan comprising a $600-\mathrm{MeV}$ linac, a $3-\mathrm{GeV}$ rapid-cycling synchrotron, and a 50-GeV synchrotron, has been officially approved for the construction of its Phase I. The Phase I includes the 1-MW pulsed spallation neutron source, but the $50-\mathrm{GeV}$ ring can be operated up to only $30 \mathrm{GeV}$. The linac energy is limited to $400 \mathrm{MeV}$, which is not high enough for the accelerator-driven nuclear waste transmutation system. The present status of the project is presented, including the result of the performance test of the already constructed components.
\end{abstract}

\section{INTRODUCTION}

The phase I of high-intensity proton accelerator facility project in Japan [1-4] was approved for the construction starting from April, 2001. The Phase I will be completed by March, 2007.

The facility comprises a $600-\mathrm{MeV}$ linac, a 3-GeV rapidcycling synchrotron (RCS), and a $50-\mathrm{GeV}$ synchrotron. A half of the $400-\mathrm{MeV}$ beams from the linac are injected to the RCS, while the other half are further accelerated up to 600 $\mathrm{MeV}$ by the superconducting (SC) linac. The RCS provides a beam power of $1 \mathrm{MW}$ to the pulsed spallation neutron experiment area with a repetition rate of $25 \mathrm{~Hz}$, while the $50-\mathrm{GeV}$ synchrotron provides a beam current of $15 \mu \mathrm{A}$ with a period of $3 \mathrm{~s}$ to either the fundamental and nuclear physics experimental area or the neutrino production target. The beams are slowly extracted to the former, while they are fast extracted to the latter.

The $600-\mathrm{MeV}$ beams from the SC linac are transported to the experimental area for the accelerator-driven nuclear waste transmutation system (ADS), where the basic study of the ADS will be conducted. The pulsed spallation neutron experiment area accommodates both the muon-production target and the neutron-production target in a series. The 10 percent of the beam power will be used for the muon production. The fundamental and nuclear physics experimental area is used for the experiments of the hypernuclei, the Kaon rare decay or others. The neutrinos produced here will be sent to the SUPERKAMIONKANDE detector located $300-\mathrm{km}$ far from the accelerator in order to do the long-base line experiment.

The facility will be constructed as a joint project of the Japan Atomic Energy Research Institute (JAERI) and the High Energy Accelerator Research Organisation (KEK). The location of the facility is the JAERI/Tokai site. The project has evolved from the Neutron Science Project (NSP) $[5,6]$ of JAERI and the Japan Hadron Facility (JHF) Project
[7-10] of KEK. The JHF project itself has evolved from the Japan Hadron Project (JHP) [11]. Now the Joint Project was approved, the detailed agreement was signed. Under this agreement the Project Team was formed in order to construct the facility. Approximately 300 staffs were assigned from the staff members of the two institutes to work as the Project Team members under the single Project Director (approximately half of them have other duties in their institutes). The Project Director placed the team members into the eight groups, including the accelerator group. The Project Team will do all the works for the construction of the facility and the research and development necessary for the project.

Since the number of accelerator staffs (109 including 5 postdoctoral fellows, and 29 staffs with other duties) are very limited, the accelerator team was organised for the highest efficiency as the following mesh structure. One grouping system is based upon the speciality: RF, vacuum, magnet, and so on. For example, the RF group is responsible for both the RF system of the $3-\mathrm{GeV}$ ring and that of the $50-\mathrm{GeV}$ ring. The vacuum group is responsible for all the vacuum system of the linac, the $3-\mathrm{GeV}$ ring and the $50-\mathrm{GeV}$ ring. On the other hand, some works should be done within a framework of each machine. Thus, the staffs are also belonging to three accelerator groups.

In the Phase I the linac will be constructed only for the RCS injection $(400 \mathrm{MeV})$. For this reason only the preliminary basic experiment is possible for the ADS. The 50-GeV synchrotron will be operated with an energy of 30 $\mathrm{GeV}$. The neutrino production target area will not be constructed in the Phase I, either. However, the full power system will be constructed for the pulsed spallation neutron source. The effort will be immediately started for the approval of the Phase II in order to start the experiments for the long-base line neutrino experiment and the ADS.

\section{LINAC}

The linac comprises a volume-production type of $\mathrm{H}^{-}$ion source, a 50-keV low-energy beam transport (LEBT), a 3$\mathrm{MeV}, 324-\mathrm{MHz}$ Radio-Frequency Quadrupole (RFQ) linac, a $50-\mathrm{MeV}$, 324-MHz Drift-Tube Linac (DTL), a 200-MeV, 324-MHz Separated DTL (SDTL), and a 400-MeV, 972MHz high-energy linac $[12,13]$. The construction of the low-energy front $60-\mathrm{MeV}$ linac [4] was already started in KEK by the JFY (Japanese Fiscal Year starting from April) 1998 supplementary budget for the JHF. In addition, some of the remaining components for the SDTL were funded by the JFY2000 supplementary budget to JAERI. All the contracts for the remaining components of the $200-\mathrm{MeV}$ 
linac will be completed by the end of JFY2001 as four-year contacts. The high-energy linac from $200 \mathrm{MeV}$ to $400 \mathrm{MeV}$ will be funded by the JFY2002 budget.

The commissioning of the 3-MeV RFQ linac has been started last March. At first we attempted the ion source without caesium, that is, purely volume production, since we prefer caesium-free ion source in order to avoid the possible decrease in the discharge limit of the following $\mathrm{RFQ}$. For this reason the peak beam current is limited to 16 $\mathrm{mA}$ so far. Further improvement of the caesium-free ion source is under way. The beam transmission through the RFQ was in agreement with the designed value.

The caesium-seeded ion source is also being developed as a back up (of course, useless, if the RFQ cannot allow the use of the caesium). After the arc discharge power supply is upgraded, the peak beam current was increased in proportion to the arc power up to $70 \mathrm{~mA}$ with an aperture size of 8 $\mathrm{mm} \phi$. The improvement in its emittance is the next step of the development, since the beam current has already exceeded the required value.

The obtainable field gradient in the SCC has been recently improved, mainly owing to the state-of-art surface electropolishing technique. Then, one can decrease the linac length for the same energy. In addition, the higher field gradient implies the larger longitudinal accepetance or the stronger longitudinal focusing, being more immune against the effect of the space charge. For these reasons we have again seriously evaluated the feasiblity of the use of the SCL from $200 \mathrm{MeV}$ to $400 \mathrm{MeV}$.

The required phase and amplitude accuracy of each cell and each tank $\left(0.1^{\circ}\right.$ and $0.1 \%$ to $1^{\circ}$ and $1 \%$, respectively, being dependent upon the kind of the errors) is much severer for the RCS injection than required just for the ADS. The deviation in $\Delta \mathrm{p} / \mathrm{p}$ should be around 0.1 percent. Therefore, the Lorentz detuning which becomes dynamic under the pulse operation should be accurately compensated. The SCC has been recently power-tested with the same pulse mode as required. The detuning is periodic from pulse to pulse. The amount of the static detuning was in agreement with the simulation [14] within a few percent. The frequency component of the dynamic detuning is mainly $50 \mathrm{~Hz}$ and $810 \mathrm{~Hz}$ with amplitudes of $4^{\circ}$ and $8^{\circ}$, respectively. The latter one was already predicted by the simulation as $765 \mathrm{~Hz}$. Its amplitude in the phase variation can be much reduced by forming the pulse shape properly.

This detuning will be accurately compensated, if one uses a system of one SC cavity per one klystron. However, a system of two SC cavities per one klystron is only competitive in cost with the normal conducting(NC) system, if one uses the SCL for the acceleration from 200 $\mathrm{MeV}$ to $400 \mathrm{MeV}$. Therefore, the feasibility of the 400$\mathrm{MeV} \mathrm{SCL}$ as an RCS injector is dependent upon how similar the detunings of the two cavities are to each other.

It is recently realized that the high field gradient imposes further severe phase-amplitude control for the same deviation of the beam energy. For the same reason as the larger acceptance, the random kick or walk and the synchrotron oscillation during the course of the accleration through the higher field gradient cavities becomes larger in the direction of the $\Delta \mathrm{p} / \mathrm{p}$ in the longitudinal phase space. Under the presence of the Lorentz detuning the field control of the SCL is obviously much harder than the NC linac. For this reason, we have finally decided to use theNC linac up to $400 \mathrm{MeV}$ as we did for the reference design.

\section{3-GEV RCS}

The construction will be started by the JFY 2002 budget. Since the Project Team is newly formed, the further optimisation of the lattice design is under way. The $3-\mathrm{GeV}$ RCS has to play two roles: the injector to the $50-\mathrm{GeV}$ synchrotron and the high-power accelerator for the spallation neutron source. This is one of the reasons for choosing the RCS rather than the full-energy linac and the storage (compressor) ring. Another reason [15] is the lower injection energy and the lower beam current of the RCS with the same beam power.. Both allow the larger beam loss during the injection process, since the radioactivity arising from the beam loss is even less than the ratio of the injection energy.

One of the most difficult problems inherent to the highenergy RCS was solved by the innovative development of the accelerating cavity loaded with magnetic alloy(MA) [16-18], one of which is FINEMET. This cavity can generate the field gradient of over $50 \mathrm{kV} / \mathrm{m}$ (potentially over $100 \mathrm{kV} / \mathrm{m}$ ) which is several times as high as conventional ferrite-loaded cavities. For this reason the RF system becomes a reasonable size even for the high-energy RCS. Further power test and beam test of the MA-loaded cavities are being continued after several successful experiments.

As an injector the $3-\mathrm{GeV}$ RCS has to match its beam longitudinally for the injection to the $50-\mathrm{GeV}$ synchrotron. For this reason the transition gamma should be much higher than $3 \mathrm{GeV}$, although the ring circumference becomes longer than the low transition gamma lattice. In addition the beam should be elongated in order to avoid a fast blow up just after the injection. Study is under way to try several methods including the phase shaking during the acceleration of the RCS.

The lattice optimisation study includes the possibility of the four-folding symmetry lattice in order to separate the scraper/collimator section from the injection section. The latter section will be thus easier to maintain, since the former section which will be very radioactive is not close to the latter. In order to keep the size of the ring as that of the reference design (three-folding symmetry) the use of the combined function magnets is seriously being considered.

\section{50-GEV SYNCHROTRON}

As mentioned in the introduction the $50-\mathrm{GeV}$ synchrotron can accelerate the beam to only $30 \mathrm{GeV}$ in the Phase I. This means that all the main lattice components are compatible 
with the beam energy of $50 \mathrm{GeV}$ in order to keep the nearfuture upgradability. The most costly component which is missing in the Phase I is a fly-wheel system for the magnet power supplies. This system is necessary for stabilising a power line down to the allowable level against its big swing due to the ramping to the $50 \mathrm{GeV}$.

All the magnets will be contracted during JFY2001 as a four-year contract. Therefore, we have only one month from this conference for the final decision on the specification of the magnets. The prototype of a full-size bending magnet has been recently produced. The field distribution measurement is under way together with its excitation curves. The newly reinforced team is further optimising the magnet design.

The RF system of the 50-GeV ring will also use cavities loaded with the same MA as that of the $3-\mathrm{GeV}$ ring. However, the $\mathrm{Q}$ value will be optimised for the $50-\mathrm{GeV}$ ring. The adjustability of the $\mathrm{Q}$ value by cutting the MA core, which is also developed for this project, is another important advantage of the MA-loaded cavity.

The slow extraction scheme is most difficult issue to solve for this kind of high-intensity, high-energy proton synchrotron. Only the one percent beam loss is allowed during the slow extraction process. An electrostatic septum ( $80 \mu \mathrm{m} \phi$ tungsten wires with rhenium) is being developed for this purpose. The voltage of $230 \mathrm{kV}$, which is higher than the necessary value of $170 \mathrm{kV}$, has been already supplied to the electrodes. Although the beam simulation results satisfy the above requirement, the further improvement in the beam loss simulation will be necessary for increasing the margin, which is needed for this kind of the beam loss/radioactivity elimination.

\section{CONCLUSION}

The high-intensity proton accelerator facility in Japan was approved for the construction at JAERI/Tokai site as a joint project of JAERI and KEK. In the Phase I, however, the linac energy is $400 \mathrm{MeV}$, while the operation energy of the $50-\mathrm{GeV}$ synchrotron is $30 \mathrm{GeV}$. The officially organised accelerator team, being just reinforced by the official start of the construction, continues the construction work already started, and began the further optimisation work of some accelerator components, if they have not yet been ordered.

\section{REFERENCES}

[1] "The Joint Project for High-Intensity Proton Accelerators", KEK Report 99-4, JHF-99-3 and JAERI-Tech 99-056 (1999).

[2] Y. Yamazaki et al., "Accelerator Complex for the Joint Project of KEK/JHF and JAERI/NSP", Proc. 1999 Part. Accler. Conf., THDL1 (1999).

[3] Y. Yamazaki et al., "High Intensity Proton Accelerators for the JAERI/KEK Joint Project", Proc. 2000 European Acceler. Conf., THOAF201(2000).

[4] Y. Yamazaki et al., "The Construction of the LowEnergy Front 60-MeV Linac for the JAERI/KEK Joint Project", Proc. 2000 Linac Conf., TUD07 (2000).

[5] M. Mizumoto et al., Proc. 1st Asian Part. Accel. Conf., 5A001-309 (1998).

[6] M. Mizumoto et al., "A High Intensity Proton Linac Development for the JAERI Neutron Science Project", Proc. 1998 Linac Conf., TU1004 (1998).

[7] JHF Project Office, "Proposal for Japan Hadron Facility," KEK Report 97-3 (JHF-97-1).

[8] JHF Project Office, "JHF Accelerator Design Study Report" KEK Report 97-16 (JHF-97-10).

[9] Y. Mori, Proc. 1997 Part. Accel.Conf., 920 (1997).

[10] Y. Yamazaki, Proc. 1st Asian Part. Accel. Conf., (1998).

[11] Y. Yamazaki and M. Kihara, "Development of the High-Intensity Proton Linac for the Japanese Hadron Project," Proc. 1990 Linac Conf., 543 (1990).

[12] T. Kato, KEK Report 96-17 (1997).

[13] Y. Yamazaki and T. Kato, Proc. 1998 Linac Conf., TU4011 (1998).

[14] N. Ouchi et al., "Development of Superconducting Cavities for High Intensity Proton Accelerator at JAERI", Proc. 1998 Applied Superconducting Conf. (1998).

[15] Y. Yamazaki, Proc. 1996 Linac Conf., 592 (1996).

[16] C. Ohmori et al., "High Field-Gradient Cavities Loaded with Magnetic Alloys", Proc. 1999 Part. Accel. Conf., THAL1 (1999).

[17] R. Muramatsu et al., "The First Beam Acceleration Test using High Gradient Cavity at HIMAC", Proc. 1999 Part. Accel. Conf., MOP59 (1999).

[18] M. Fujieda et al., "Magnetic Alloy Loaded RF Cavity for Barrier Bucket Experiment at the AGS", Proc. 1999 Part. Accel. Conf., MOP81 (1999). 